standardisation. They have the advantage of enabling the mortality or incidence of a disease to be compared between groups with different age structures but they allow for the effect that this difference will have on the comparison. Nevertheless, these ratios also have the disadvantage common to all summary statistics: they may conceal subtle, but possibly important, differences in the relation of the rates with age itself.

An example discussed recently is the comparison of the male standardised mortality ratio for two diseases in different counties in England and Wales and in different groups classified by their smoking habit ( $R$ West, unpublished data). ${ }^{1}$ For lung cancer and ischaemic heart disease there is a familiar association of high (age standardised) death rates with high consumption of cigarettes. Nevertheless, the pattern of death rates by age according to the amount smoked is obviously different. For lung cancer there is an increasing divergence as age increases, in contrast to ischaemic heart disease, for which the age specific mortality appears merely to be displaced horizontally (figure). Thus the author, West, seeks to distinguish between a genuinely higher risk of death at a given age and merely a reduction in the average age of death, which will, of course, look like a higher age specific risk if the disease is associated with an increasing risk with age.

The point of this distinction becomes clearer when West compares the age specific risk of death from all causes with whether the subjects smoked or not. These graphs resemble those for ischaemic heart disease, not those for lung cancer. Since all men eventually die the interpretation then cannot be that smoking causes more deaths than not smoking but that, on average, smoking brings forward the age of death. Similarly, therefore, does smoking merely bring forward the age of death from ischaemic heart disease? In contrast, smoking genuinely does cause many more deaths from lung cancer than occur among non-smokers.

The argument is made more cogent when comparisons of mortality are attempted between the counties of England and Wales. Those counties with highest standardised mortality ratios for ischaemic heart disease are not necessarily those in which the age standardised proportions of all deaths from ischaemic heart disease are highest. Indeed, comparing the counties with the highest standardised mortality ratios with the ones with the lowest suggests that the proportions of all deaths from ischaemic heart disease at ages above about $\mathbf{5 0}$ are similar.

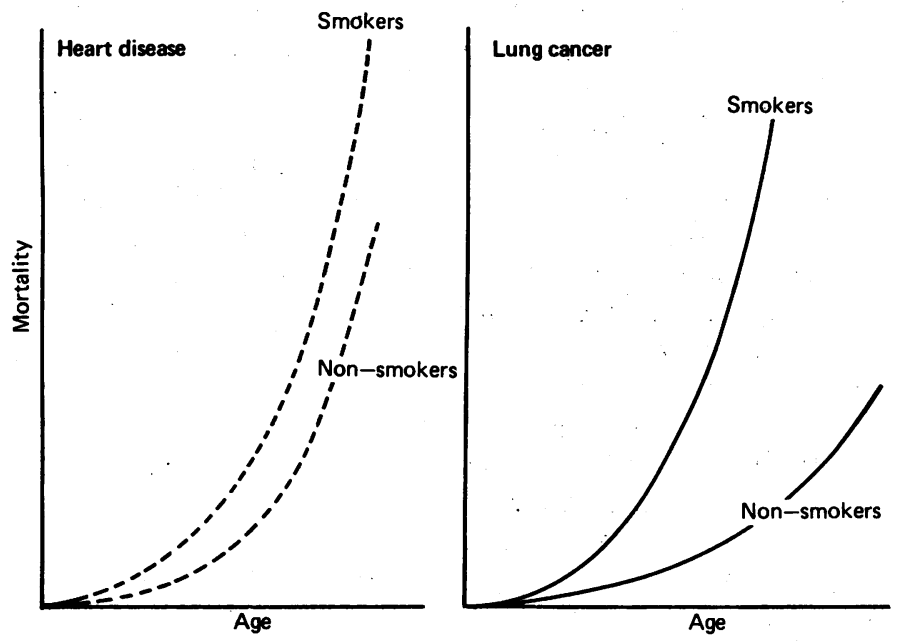

Variation in mortality from lung cancer and ischaemic heart disease with age in smokers and non-smokers.
This is pertinent because in epidemiology we tend to treat all diseases similarly. Thus a relatively high age standardised rate of one disease among a group of people will tend to trigger a search for a risk factor to which this group is particularly exposed. But in this example spotting a high standardised mortality ratio for lung cancer would mean that an epidemiologist stood more chance of finding the risk factor than spotting a high standardised mortality ratio for ischaemic heart disease-because the high standardised mortality ratio for lung cancer reflects more deaths relative to the whole country, whereas the high standardised mortality ratio for ischaemic heart disease may reflect only earlier deaths. Thus for lung cancer a raised standardised mortality ratio could reflect exposure to a risk factor, whereas the equally high standardised mortality ratio for ischaemic heart disease may not.

Thus West makes important distinctions for interpreting के high (or low) standardised death (or incidence) rates for $\overrightarrow{0}$ particular diseases. Firstly, do they reflect more deaths or merely the same lifetime risk of death brought forward? To $\vec{\omega}$ make this distinction clearer the researcher must look at all cause mortality. Since everybody dies, differences in overall death rates can only mean younger deaths. If, secondly, the proportion of cause specific deaths of all deaths remains roughly constant then looking for a particular risk factor may be futile despite a raised cause specific standardised mortality ratio. This is because the particular cause of death may be merely one reflection of earlier deaths in general that may be a consequence of many factors.

KLIM MCPHERSON

University Lecturer in Medical Statistics,

Department of Community Medicine and General Practice,

Radcliffe Infirmary,

Oxford OX2 6HE

1 West RR. High death rates: more deaths or earlier deaths? $\mathcal{F} R$ Coll Physicians Lond 1987;21:73-6.

\section{Back calculation of blood alcohol concentration}

In July Lord Justice Watkins ruled that it was legally permissible to back calculate to the time of an accident the blood alcohol concentrations in motorists suspected of drink-driving offences. His judgment was that the law places $\delta$ no restriction on the method of establishing the blood alcohol concentration, but he did express caution as to its practic- $\frac{9}{7}$ ability. Dr John Havard, secretary of the BMA, has described $N$ the procedure as unscientific, and some consider that a calcu- $D$ lation based on a single sample is scientifically unacceptable. 을.

Scientific predictions are made in terms of probability, $\tilde{N}$ calculated by statistical procedures, and based on multiple measurements to take account of biological variation. The $\underset{\sigma}{\sigma}$ law functions similarly, dealing in terms of "reasonable $\theta$ doubt," and so, unusual circumstances apart, back calcu- $\underset{\varnothing}{\mathscr{D}}$ lation could prove acceptable.

Alcohol disappears from the blood in a (pseudo) linear manner. ${ }^{1}$ Not only, however, is there biological variability in the rate of decline of the blood alcohol concentration among individuals and on different occasions but also the rate varies considerably with the dose of alcohol..$^{2-4}$ Studies have provided upper and lower limits for the rate of decline in different sorts of drinkers: social drinkers 19 and $11 \mathrm{mg} / 100 \mathrm{ml} / \mathrm{h}$, non- 
drinkers 16 and 8 , alcoholics 39 and 21 (social drinkers $4 \cdot 1$ and $2.4 \mathrm{mmol} / \mathrm{l} / \mathrm{h}$, non-drinkers 3.5 and 1.7 , alcoholics 8.5 and 4.6). ${ }^{56}$ But probably these values do not apply to all groups in the community. Back calculation along these extremes of slope gives the range of blood alcohol concentration at the time of the accident that might have led to the measured value. This range can be narrowed by adjusting for the person's weight, build, age, sex, and body fat.

Even with several timed blood specimens, however, the standard slope still has to be used because present assays are not precise enough to calculate accurately the person's own slope. ${ }^{47}$ There are also serious constraints on the timings between which the back projections can be made. These arise because the blood alcohol concentration is not predictable when absorption is still occurring ${ }^{8}$ and because the slope departs appreciably from linearity at low alcohol concentrations. When some $30 \mathrm{~g}$ of alcohol is drunk over a short period ( 5 minutes) on an empty stomach the blood concentration rises quickly to produce a peak between 30 and 60 minutes; then the concentration declines in a near linear fashion for about four hours. When the concentration approaches 20 $\mathrm{mg} / 100 \mathrm{ml}(4.3 \mathrm{mmol} / \mathrm{l})$ the curve becomes non-linear and back extrapolation is invalid. If the same quantity of alcohol is taken slowly and with food the blood concentration rises less sharply to a more rounded peak and gradually turns into a linear downward slope after about three hours as the rate of absorption falls below the removal rate. During this absorption phase the blood alcohol concentration may be much lower than when the alcohol is drunk rapidly, and it is not valid to extrapolate back into this absorption phase from the subsequent linear slope. When alcohol has been drunk after an accident ("the hip flask defence") attempts to allow for this require either an estimate or "calibration" of the person with alcohol, 9 but this calibration may be invalid if measurements are delayed for many weeks or months.

Where it can be verified that a motorist of normal physique has not had alcohol in the previous two to three hours then determination of the blood alcohol concentration by back calculation in the following two to three hours would provide a range of concentrations-albeit with wide confidence intervals - that are likely to be right. Additional evidencefor instance, that the person did not take food with the drink, on the amount drunk, and on when it was drunkwill extend the valid "window" to an earlier time. But even with extra evidence it is difficult to be confident of blood alcohol concentrations in the hour after drinking - and this is the time when most accidents are likely to have happened.

K O LEWIS

Principal Biochemist,

Department of Clinical Chemistry,

Dudley Road Hospital,

Birmingham B18 7QH

1 Widmark EMP. Verteilung und Umwandlung des Athylalkohols im Organismus des Hundes Biochemische Zeitschrift 1933;267:128-34.

2 Newman HW, Lehman AJ, Cutting WC. Effect of dosage on rate of disappearance of alcohol from the blood stream. I Pharmacol 1937;61:58-61.

3 Wagner JG. Properties of the Michaelis-Menten equation and its integrated forms which are useful in pharmacokinetics. F Pharmacokinet Biopharm 1973;1:103-21

4 Wagner JG, Wilkinson PK, Sedman AJ, Kay DR, Weidler DJ. Elimination of alcohol from human blood. I Pharm Sci 1976;65:152-4.

5 Winek CL, Murphy KL. The rate and kinetic order of ethanol elimination. Forensic Sci In 1984;25:159-66.

6 Lewis MJ. Blood alcohol: the concentration time curve and retrospective estimation of level. I Forensic Sci Soc 1986;26:95-113.

7 Forrest ARW. Non-linear kinetics of ethyl alcohol metabolism. F Forensic Sci Soc 1986;26:121-3. 8 Madsen BW, Rossi L. Sleep and Michaelis-Menton elimination of ethanol. Clin Pharmacol Ther 1980;27:114-9.

9 Lewis MJ. The individual and the estimation of his blood alcohol concentration from intake, with particular reference to the "hip-flask" drink. I Forensic Soc 1986;26:19-27.

\section{Radiological diagnosis of deep vein thrombosis}

The clinical evaluation of deep vein thrombosis is very inaccurate. Some two thirds of thromboses in the legs cause no symptoms and half the diagnoses made on clinical grounds alone will be wrong. ${ }^{1}$ Hence it is important to evaluate the other methods of diagnosis.

Venography of the legs remains the gold standard against which all other methods have to be judged. The introduction of low osmolar contrast media has greatly reduced the upsets caused by venography, ${ }^{23}$ but a search has also been made for methods which are less invasive and avoid the use of $x$ rays. Both impedance plethysmography and Doppler ultrasonography are highly sensitive in diagnosing occlusive deep vein thrombosis in the iliac and femoral veins but are much less so in detecting thrombosis in the lower leg as well as the potentially dangerous proximal non-occlusive thrombosis. ${ }^{45}$ "Duplex" ultrasonic scanning - that is, the combination of pulsed Doppler and real time B mode imaging-requires expensive equipment and has not been evaluated in patients with suspected deep vein thrombosis. Radionuclide imaging using fibrinogen labelled with iodine-125 requires at least 2448 hours before a diagnosis can be made and cannot be used in pregnant or lactating women; it is sensitive in detecting thrombi in the calf but less so in the proximal veins. ${ }^{6}$ Technetium- $99 \mathrm{~m}$ has been tagged to fibrinogen and other blood products, ${ }^{7}$ but imaging using these combinations has not been as accurate as venography.

Recently real time B mode ultrasound scanning, usually with a $7.5 \mathrm{MHz}$ transducer, has been evaluated for confirming or excluding deep vein thrombosis. It is quick, it uses standard equipment that is available in most $x$ ray departments, and many radiologists are skilled in ultrasonic scanning. The results from different centres have been remarkably consistent: a specificity of $89-94 \%$ and a sensitivity of $100 \%$. $^{8-11}$ The visualisation of thrombus is the most specific diagnostic criterion. ${ }^{8}$ Acute and chronic thrombi can often be distinguished by ultrasound: typically the latter are highly echogenic, ${ }^{12}$ and the former are not. ${ }^{13}$ Finding clot floating free in the lumen of the vein is further evidence of recent thrombosis ${ }^{8}$ and compressibility of the vein by the ultrasound pulse is another sensitive and reproducible indicator. ${ }^{11} 13$ The common femoral vein may fail to distend in response to the Valsalva manoeuvre in ileofemoral venous occlusion. ${ }^{14}$ Venous compression should distinguish the slow moving echoes which arise in the lumen from venous stasis caused by thrombosis higher in the vein, cardiac failure, or ischaemia of the leg..$^{15}$

Real time scanning of the venous system cannot show up the calf veins (except occasionally for their proximal portions) and the iliac veins. ${ }^{11}$ Another blind area on ultrasonography is the popliteal-femoral segment in the region of the adductor opening. ${ }^{10}$ Nevertheless, most workers consider that thrombi limited to the calf veins are unlikely to give rise to embolism, ${ }^{16}$ and they often resolve within 72 hours without anticoagulant treatment. ${ }^{17}$

So what conclusions can we reach about the roles of ultrasonography and venography in practice? Given a radiologist skilled in ultrasonography, this method is an accurate way of excluding or confirming thrombus in the femoropopliteal segment. When a radiologist does not have this skill venography of the leg remains a first line investigation for suspected thrombosis there and in the iliac veins. An 